\title{
2-D Generating Function of the Zernike Polynomials and their Application for Image Classification
}

\author{
Barmak Honarvar Shakibaei Asli ${ }^{1,2, *}$, Jan Flusser ${ }^{1,3}$ and Yifan Zhao ${ }^{2}$ \\ ${ }^{1}$ Czech Academy of Sciences, Institute of Information Theory and Automation, \\ Pod vodárenskou věží 4, 18208 Praha 8, Czech Republic \\ e-mail: \{honarvar,flusser\}@utia.cas.cz \\ 2 Through-Life Engineering Services Centre, School of Aerospace, Transport and Manufacturing, \\ Cranfield University, Cranfield, Bedfordshire MK43 0AL, UK \\ e-mail: \{barmak,yifan.zhao\}@cranfield.ac.uk \\ 3 Faculty of Management, University of Economics, Jindřichuv Hradec, Czech Republic \\ * Barmak Honarvar Shakibaei Asli: Corresponding author
}

\begin{abstract}
This work proposes a new approach to find the generating function (GF) of the Zernike polynomials in two dimensional form. Combining the methods of GFs and discretetime systems, we can develop two dimensional digital systems for systematic generation of entire orders of Zernike polynomials. We establish two different formulas for the GF of the radial Zernike polynomials based on both the degree and the azimuthal order of the radial polynomials. In this paper, we use four terms recurrence relation instead of the ordinary three terms recursion to calculate the radial Zernike polynomials and their GFs using unilateral 2D Z-transform. A spatio-temporal implementation scheme is developed for generation of the radial Zernike polynomials. Since Zernike moments (ZMs) are invariant with respect to rotation, translation and scaling, the experimental schemes show the image classification applications by using the proposed algorithm.
\end{abstract}

Keywords-Optics; Zernike polynomials; Image classification; Generating function; Z-transform.

\section{INTRODUCTION}

Zernike polynomials are a set of basis functions that satisfy the orthogonality property on the continuous unit disk [1]. This set has found numerous applications in a variety of fields: optics [2], wavefront sensing [3], [4], aberration theory [4], scaled pupils [5], [6], [7], adaptive optics [8], highresolution optical wave-front control system [9], and image processing [10], [11]. However, direct computation of the Zernike polynomials, especially calculation of the factorial terms in the radial component, is time consuming. Many methods have been developed to speed up the computation of Zernike radial polynomials [12], [13], [7]. Recently, in [14], a new approach was extended to compute the radial Zernike polynomials using four term recurrence relation. Unlike the previous algorithms, the derived recurrence relation depends neither on the degree nor on the azimuthal order of the radial polynomials. This leads to a reduction in the computational complexity.

Since the radial Zernike polynomials play an important role in the domain of optics and image processing, there is a connection between these polynomials and their GF to develop an analytical study for designing a filter-generating
Zernike system. Born and Wolf introduced the GF of the radial Zernike polynomials using their relation with the Jacobi polynomials [15]. This GF is only based on the degree of the radial polynomials and the azimuthal order of the radial polynomials is still apparent in the derived GF.

In the engineering literature, especially in the area of digital signal processing, the transfer function is spoken of as the Ztransform of the unit-sample response. The generating function and the Z-transform are similar but different. They both generate the same causal sequence.

In robotics and computer vision, Zernike moments play an important role as shape descriptors for object recognition [16], [17]. Zernike moments are projections of the image function onto a set of Zernike polynomials. Magnitudes of Zernike moments possess the invariance with respect to object rotation, which is their main advantage.

In this paper, we firstly generalize the definition of the GFs for two-variable Zernike polynomials, then derive a simple method to obtain the two-variable GF of these polynomials by using a four term recurrence relation and Z-transform application. Finally, we design a filter-generating Zernike system which is a very efficient tool for systematic generation of the radial Zernike polynomials with different degrees and azimuthal orders. This is the motivation for us to present a new type of the GF for two-variable Zernike polynomials for designing of a filter-based system.

\section{Revisiting Generating Functions}

Generating functions (GFs) are a powerful tool as in the theory of classical orthogonal polynomials, as in various applications [18]. There are many types of GFs, including ordinary GFs, exponential GFs, Lambert series, Bell series, and Dirichlet series. Polynomials are a special case of ordinary GFs, corresponding to finite sequences that vanish after a certain point. These are important in that many finite sequences can usefully be interpreted as GFs. The ordinary GF of a 
sequence $a_{n}$ is

$$
G\left(a_{n} ; u\right)=\sum_{n=0}^{\infty} a_{n} u^{n} .
$$

This function can be generalized to arrays with multiple indices. For example, the ordinary GF of a two-dimensional array $a_{m, n}$ can be defined as

$$
G\left(a_{m, n} ; u, v\right)=\sum_{n=0}^{\infty} \sum_{m=0}^{\infty} a_{m, n} u^{n} v^{m} .
$$

\section{A. Applications of $G F s$}

Working with a continuous function is sometimes much easier than working with a discrete function (sequence). For example, in the analysis of functions, calculus is very useful. However, the discrete nature of sequences prevents us from using calculus on sequences. A GF is a continuous function associated with a given sequence. For this reason, GFs are very useful in analyzing discrete problems involving sequences of numbers or sequences of functions. Many combinatorial problems can be solved with the aid of GFs. Therefore, GFs are powerful because they allow us to use tools from calculus and analysis to solve problems in areas such as discrete mathematics and combinatorics, where such tools do not seem readily applicable. They are useful in proofs and convolutions of series, e.g. time series and signal processing ideas can be seen in terms of GFs. There are quite a few applications, especially considering how much they are used in statistical and mathematical theory.

\section{B. Z-transform method to find the GF}

Revisiting Eqs. (1) and (2), reminds us the schema of Ztransform. By replacing $u=z^{-1}$ in 1D form or $u=z_{1}^{-1}$ and $v=z_{2}^{-1}$ in $2 \mathrm{D}$ form and recalling the unilateral $1 \mathrm{D} / 2 \mathrm{D} \mathrm{Z}$ transform of sequences $a_{n}$ or $a_{m, n}$, it is easy to find a closed form for the left hand side of (1) or (2) as one/two-variable GFs. To find GFs of a sequence or polynomial, it is possible to make a bridge from recursion to GF.

As a very simple example, let us consider the well known Fibonacci polynomials. These Fibonacci polynomials are defined by a recurrence relation:

$$
\begin{aligned}
& F_{n+2}(x)=x F_{n+1}(x)+F_{n}(x), \quad n \geq 0 \\
& \text { with } F_{0}(x)=0 \text { and } F_{1}(x)=1 .
\end{aligned}
$$

There are time-shifting properties of the unilateral Z-transform for the first and the second time-advance as follow:

$$
\begin{aligned}
f(n) & \stackrel{\mathscr{Z}}{\longleftrightarrow} F(z), \\
f(n+1) & \stackrel{\mathscr{Z}}{\longleftrightarrow} z(F(z)-f(0)), \\
f(n+2) & \stackrel{\mathscr{Z}}{\longleftrightarrow} z^{2}\left(F(z)-f(0)-f(1) z^{-1}\right) .
\end{aligned}
$$

By applying the Z-transform to (3) with respect to discrete variable $n$, we get

$$
\begin{aligned}
z^{2}\left(F(x ; z)-F_{0}(x)-F_{1}(x) z^{-1}\right) & =x z\left(F(x ; z)-F_{0}(x)\right) \\
& +F(x ; z)
\end{aligned}
$$

and, finally by substituting the initial values, $F_{0}(x)=0$, $F_{1}(x)=1$ and $u=z^{-1}$ into (5) and some simplification, we arrive at

$$
F(x ; u)=\frac{u}{1-x u-u^{2}} .
$$

Eq. (6) expresses the ordinary GF of Fibonacci polynomials. It can be comparable with the second order "all-pole" filtergenerating system and operates on polynomial signal in indeterminate $z^{-1}$ as $\frac{z^{-1}}{1-x z^{-1}-z^{-2}}$. The filter-generating system for this example of Fibonacci polynomials is excited by an impulse signal under the condition of zero initial states in (3) and can be implemented as a direct-form structure in Fig. 1. This figure shows a second order infinite impulse response (IIR) filter block diagram. Each delay block represents a unit delay which equals to term $z^{-1}$. In other words, the filtergenerating system can be viewed as a 1D causal IIR system that, when excited by a 1D impulse signal, $\delta(n)$, generates the impulse response of the $n$th digit of the family at the $n$th point of its $1 \mathrm{D}$ impulse response. However, using this Z-

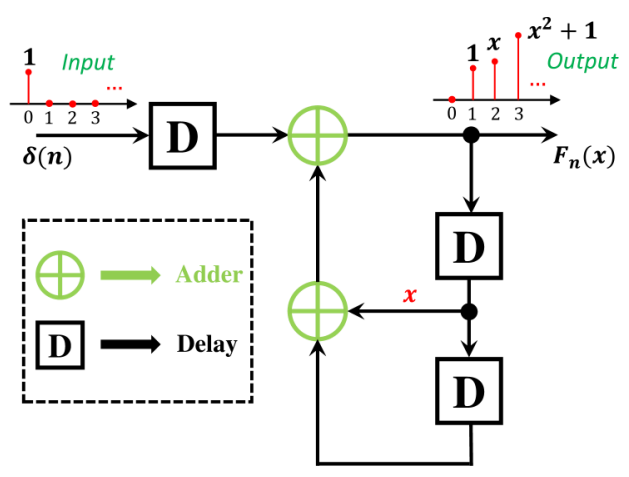

Fig. 1: Direct-form recursive structure for Fibonacci rational filter-generating system.

transform method, it is possible to find the GFs through a three-term recurrence of polynomials or sequences. In some rare cases such as the aforementioned example, the coefficients of the recurrence relation do not depend on the order of the polynomial $(n)$ which makes the Z-transform easy. While, in many cases such as Hermite, Chebyshev, Legendre, etc., the coefficients of three-term recurrence depend on the order of polynomials that needs to use more properties of Z-transform to derive the GF. We use the same algorithm to find the GF of the complicated Zernike polynomials in 2-D format using an easy four-term recurrence explained in the next section. This leads us to develop a spatio-temporal implementation scheme for generation of the radial Zernike polynomials. 


\section{Proposed Method to Obtain GF of the Zernike POLYNOMIALS}

This section focuses on the four-term recurrence of the radial Zernike polynomials instead of their three-term recurrence relations to reach a simple solution for deriving the GF [15].

\section{A. Zernike Polynomials}

The Zernike polynomials are a set of orthogonal polynomials that arise in the expansion of a wavefront function for optical systems with circular pupils. They are a sequence of polynomials that are orthogonal on the unit disk. Named after optical physicist Frits Zernike, winner of the 1953 Nobel Prize in Physics and the inventor of phase-contrast microscopy, they play an important role in beam optics [1]. In general, Zernike polynomials are defined in polar coordinate $(r, \theta)$ as

$$
Z_{n}^{m}(r, \theta)=R_{n}^{m}(r) e^{-j m \theta},
$$

where $R_{n}^{m}(r)$ are the radial polynomials, $n$ is the degree and $m$ is the azimuthal order of these polynomials (both are nonnegative integers with $n \geq m)$. $R_{n}^{m}(r)$ can be defined as follows:

$$
R_{n}^{m}(r)=\sum_{k=0}^{\frac{n-|m|}{2}}(-1)^{k} \frac{(n-k) !}{k !\left(\frac{n+m}{2}-k\right) !\left(\frac{n-m}{2}-k\right) !} r^{n-2 k} .
$$

The radial Zernike polynomials have the GF in 1D form [15]

$$
\sum_{n=0}^{\infty} R_{m+2 n}^{ \pm m}(r) u^{n}=\frac{\left(1+u-\sqrt{1+2 u\left(1-2 r^{2}\right)+u^{2}}\right)^{m}}{(2 r u)^{m} \sqrt{1+2 u\left(1-2 r^{2}\right)+u^{2}}} .
$$

As can be seen from the above equation, the GF still depends on the azimuthal order, $m$, which can be eliminated by using the definition of two-variable GFs (see subsection III-C).

Notice that we do not apply the geometric progression to Eq. (9) to generalize 1D form of GF to derive 2D form of its GF since the common ratio of this geometric progression is not always less than one while in the definition of 2D GF, there is no limitations for variables $u$ and $v$ (see Eq. (2)).

The Zernike polynomials satisfy the following four-term recurrence relation which depends neither on the degree nor on the azimuthal order of the radial polynomials [14]:

$$
R_{n}^{m}(r)+R_{n-2}^{m}(r)=r\left(R_{n-1}^{|m-1|}(r)+R_{n-1}^{m+1}(r)\right) .
$$

By using (7) in (10), four-term recurrence relation can be generalized for the Zernike polynomials as:

$$
\begin{aligned}
Z_{n}^{m}(r, \theta)+Z_{n-2}^{m}(r, \theta)= & r\left(Z_{n-1}^{|m-1|}(r, \theta) e^{j \theta}\right. \\
& \left.+Z_{n-1}^{m+1}(r, \theta) e^{-j \theta}\right) .
\end{aligned}
$$

It should be noted that, according to the traditional recursion of Zernike polynomials, all of them depend on degree $n$ or both degree $n$ and azimuthal order $m$ [13].
Fig. 2 shows a block diagram for generating the radial Zernike polynomials in a right triangle form. In this triangle form, we distinguish three cases: (a) the radial Zernike polynomials which might be obtained on the hypotenuse of triangle. All the radial Zernike polynomials over this line obey $n=m$, (b) all the radial Zernike polynomials that have been illustrated in the left side cathetus of triangle have zero azimuthal order; $m=0$, and (c) the rest of the radial Zernike polynomials generated inside of this triangle between hypotenuse and the left side cathetus obey $n>m, m \neq 0, n \neq m$.

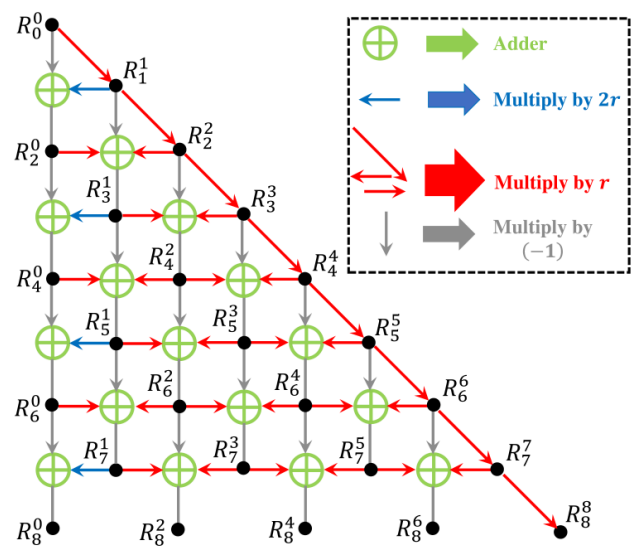

Fig. 2: Generating system block diagram of the radial Zernike polynomials.

\section{B. Implementation of recursive generating system of the radial Zernike polynomials}

A spatio-temporal scheme is a method for filtering digital signals through the filter-generating system that generates array structures for the family of filters [19]. In this scheme, we consider recursive filter-generating system that provides a simple and systematic method for deriving each term of intended structure. The explained recursion in (10) can be divided into three cases as mentioned in previous subsection. Fig. 3 shows three cases based on simplification of (10) for (a) $n=m$, (b) $m=0$, and (c) $n>m, m \neq 0, n \neq m$. As we see in this figure, the first case requires only one multiplier when $n=m$. The second case requires two multipliers and one adder whether the third case requires two multipliers and two adders. We show multipliers by $\mathbf{M}$ and the adders by A. Therefore, we present three blocks to generate the radial Zernike polynomials with different degrees and azimuthal orders.

Based on three cases in Fig. 3 and using the generating system block diagram of the radial Zernike polynomials which is shown in Fig. 2, it is possible to implement a cascaded filter form of the radial Zernike polynomials. Fig. 4 illustrates such a network by using cascaded blocks: 1M, 1A $2 \mathrm{M}$ and $2 \mathrm{~A}$ $\mathbf{2 M}$. The first row of this lattice generates the radial Zernike polynomials with $n=m$ using cascaded blocks of $\mathbf{1 M}$. The left column generates those polynomials with $m=0$ 


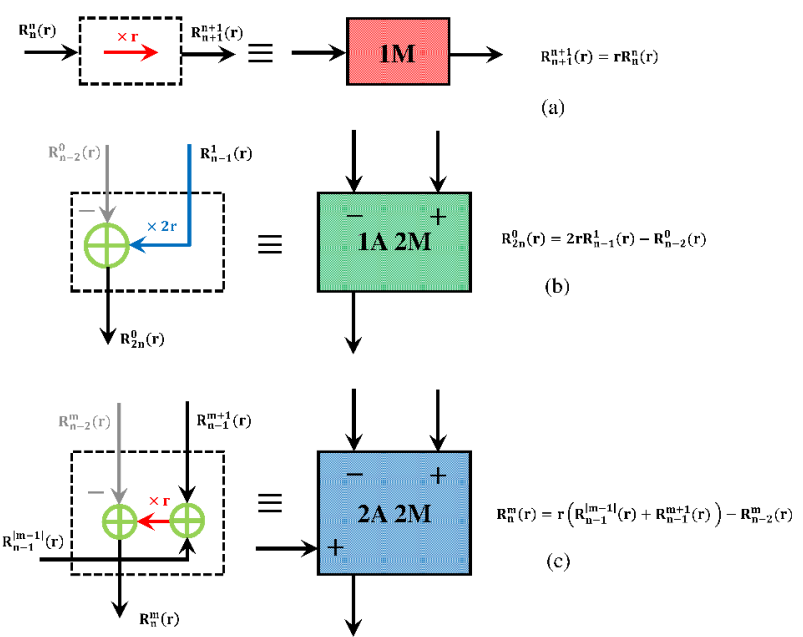

Fig. 3: Spatio-temporal implementation of recursive generating system of the radial Zernike polynomials using adders and multipliers.

using 1A 2M blocks whether the rest of lattice works for $n>m, m \neq 0, n \neq m$ using $\mathbf{2 A} \mathbf{2 M}$ blocks.

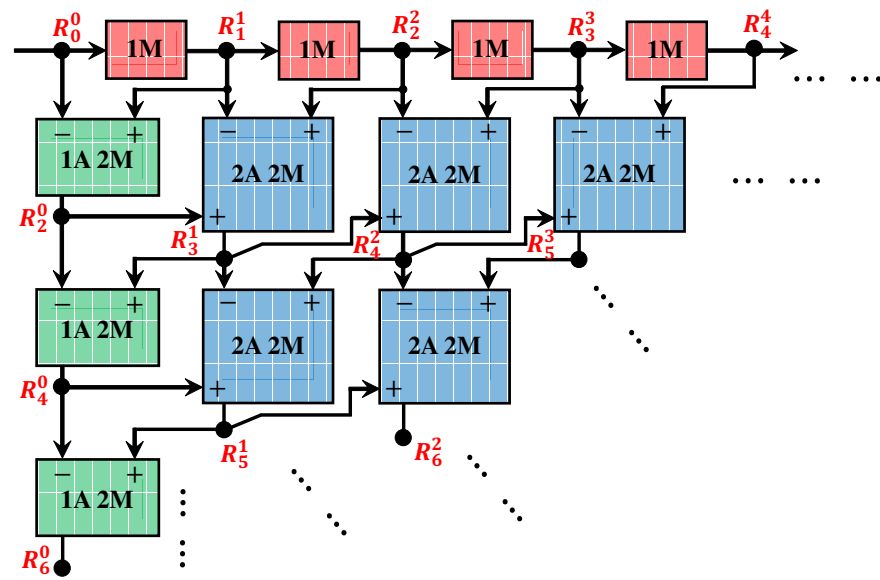

Fig. 4: Zernike network: A cascaded lattice to generate the radial Zernike polynomials.

\section{Deriving the Proposed GF of the radial Zernike Polyno- mials}

As we mentioned in subsection II-B, by applying the Ztransform method on a recurrence relation, it is possible to get the GF of a polynomial/sequence. We would use the $2 \mathrm{D}$ Z-transform of the radial Zernike polynomials to obtain the $\mathrm{GF}$ of them with two-variables as:

$$
G\left(R_{n}^{m}(r) ; u, v\right)=\sum_{n=0}^{\infty} \sum_{m=0}^{\infty} R_{n}^{m}(r) u^{n} v^{m} .
$$

Note that in the above equation, if we consider $u$ as $z_{1}^{-1}$ and $v$ as $z_{2}^{-1}$, it can be described as a 2D Z-transform of the radial Zernike polynomials of degree $n$ and the azimuthal order $m$.
By changing $m$ to $m+1$ and $n$ to $n+2$ in (10), we get:

$$
R_{n+2}^{m+1}(r)+R_{n}^{m+1}(r)=r\left(R_{n+1}^{m}(r)+R_{n+1}^{m+2}(r)\right) .
$$

Equation (13) is a second order time-advance difference equation in terms of $n$ and $m$. It is clear that we can find the similar time-shifting properties of the unilateral 2D Z-transform of this equation as Eqs. (4). To do so, each term of the four-term recurrence relation of the radial Zernike polynomial should be transformed to the Z-domain using time-shifting properties of the 2D unilateral Z-transform. The generalized form of Eqs. (4) can be applied for the first and the second time-advance properties of 2D unilateral Z-transform. For example, to find the 2D Z-transform of the first term in (13), we need to use the 2D Z-transform time-advance properties as follow:

$$
\begin{aligned}
\mathscr{Z}\left\{R_{n+2}^{m+1}(r)\right\} & =z_{1}^{2} z_{2}\left[R\left(r ; z_{1}, z_{2}\right)-\sum_{m=0}^{\infty} R_{0}^{m}(r) z_{2}^{-m}\right. \\
& -\sum_{n=0}^{\infty} R_{n}^{0}(r) z_{1}^{-n}-\sum_{m=0}^{\infty} R_{1}^{m}(r) z_{1}^{-1} z_{2}^{-m} \\
& \left.+R_{0}^{0}(r)+R_{1}^{0}(r) z_{1}^{-1}\right] .
\end{aligned}
$$

The first summation is only valid for $m=0$ that makes it 1 . The second summation can be obtained from the $1 \mathrm{D}$ generating function of the radial Zernike polynomial in [15] that might be simplified using (9) since $m=0$ which leads it to (set $\left.u=z^{-1}\right)$ :

$$
\sum_{n=0}^{\infty} R_{2 n}^{0}(r) z^{-n}=\frac{1}{\sqrt{1+2 z^{-1}\left(1-2 r^{2}\right)+z^{-2}}} .
$$

The third summation is only valid for $m=1$ that makes it $r z_{1}^{-1} z_{2}^{-1}$. Finally, the forth and fifth terms are approaching to 1 and 0 , respectively according to definition of the radial Zernike polynomials. By rewriting (14) based on these arguments one obtains:

$$
\begin{aligned}
\mathscr{Z}\left\{R_{n+2}^{m+1}(r)\right\} & =z_{1}^{2} z_{2}\left[R\left(r ; z_{1}, z_{2}\right)\right. \\
& \left.-\frac{1}{\sqrt{1+2 z_{1}^{-1}\left(1-2 r^{2}\right)+z_{1}^{-2}}}\right]-r z_{1} .
\end{aligned}
$$

We can do the same procedure for the remaining terms of Eq. (13) to find the 2D unilateral Z-transform of them. Then by taking the unilateral Z-transform of Eq. (13) and using the aforementioned 2D Z-transforms, we can obtain:

$$
\begin{aligned}
& R\left(r ; z_{1}, z_{2}\right) \\
& =\frac{2+z_{1}^{2}\left(1+\sqrt{1+2 z_{1}^{-1}\left(1-2 r^{2}\right)+z_{1}^{-2}}\right)-z_{1}\left(1+2 r z_{2}\right)}{2 \sqrt{1+2 z_{1}^{-1}\left(1-2 r^{2}\right)+z_{1}^{-2}}\left(1-r z_{1}\left(z_{2}+z_{2}^{-1}\right)+z_{1}^{2}\right)} .
\end{aligned}
$$

To obtain the two-variable GF of the radial Zernike polyno- 
mial, we can change $z_{1}^{-1}$ to $u$ and $z_{2}^{-1}$ to $v$ as:

$$
\begin{aligned}
G & \left(R_{n}^{m}(r) ; u, v\right) \\
& =\frac{2 u^{2} v+v\left(1+\sqrt{1+2 u\left(1-2 r^{2}\right)+u^{2}}\right)-u(v+2 r)}{2 \sqrt{1+2 u\left(1-2 r^{2}\right)+u^{2}}\left[v\left(1+u^{2}\right)-r u\left(1+v^{2}\right)\right]} .
\end{aligned}
$$

\section{Generalization: GF of the Complex Zernike Polynomials}

In this subsection, a generalized GF for the complex Zernike polynomials is derived. Since Eq. (11) is the generalized form of (10), it is possible to do the similar derivation of GF for it. The result can be written as:

$$
\begin{aligned}
G\left(Z_{n}^{m}(r, \theta) ; u, v\right) & =\frac{2 v\left[1+u^{2}+r A(u) u v\left(1-e^{j \theta}\right)\right]}{2 A(u)\left[v\left(1+u^{2}\right)-r u\left(e^{-j \theta}+v^{2} e^{j \theta}\right)\right]} \\
& +\frac{[v(A(u)-1)-u(v+2 r)] e^{-j \theta}}{2 A(u)\left[v\left(1+u^{2}\right)-r u\left(e^{-j \theta}+v^{2} e^{j \theta}\right)\right]},
\end{aligned}
$$

where $A(u)=\sqrt{1+2 u\left(1-2 r^{2}\right)+u^{2}}$. It is clear from (19) that by assuming $\theta=0$, this equation will be shortened to (18). The derived GFs of the radial Zernike polynomials in 2D form show their compatibilities with 2D discrete variables of the Zernike polynomials $(n, m)$. Born and Wolf expressed the 1D form of the radial Zernike polynomials' GF while the azimuthal order $m$ still exists in this formula [15].

\section{EXPERIMENT}

To illustrate the practical usefulness of the proposed algorithm, we performed the following experiment motivated by tasks one has to resolve in autonomous robot navigation.

The movement and actions of autonomous robots is often controlled by external navigation marks, that are placed in the environment the robot moves through. Each mark may have encoded instructions for the robot. The robots have to scan its visual field, look for the marks, recognize them and then proceed as required. The marks may be simple patterns with specified meaning of each mark type. The challenge is that the visual recognition system should be robust to various orientation, size and viewing angles under which the marks appear in the visual field of the robot. Zernike moments (ZMs) are useful features for the mark recognition, because their magnitudes are invariant to rotation and thanks to the mapping onto a unit circle they are also scale invariant [20]. They are defined as projections onto Zernike polynomials

$$
A_{n m}=\frac{n+1}{\pi} \int_{0}^{2 \pi} \int_{0}^{1} Z_{n}^{m}(r, \theta) f(r, \theta) r \mathrm{~d} r \mathrm{~d} \theta,
$$

where $Z_{n}^{m}(r, \theta)$ is defined in (7) and $f(r, \theta)$ is the image function in polar coordinate. Since the recognition should be real-time, an efficient algorithm for ZM computation is highly desirable.

We performed the experiment in a real indoor environment using the digits $0,1,2, \ldots 9$ as the navigation marks. We mounted the printed digits in a picture frame which was placed near to the corner and furniture of the room (see Fig. 5 for an example).

We intentionally put some of them in random orientations to make the test more challenging. Since we did not have an actual robot, we took pictures by a hand-held camera, without paying much attention to camera position, set-up and viewing angle; exactly as a real robot would do. In this way, we obtained three query images of unknown digits. We segmented and extracted the digits from the images, calculated ZMs up to the 4th order using the proposed algorithm and classified the digits against the database of the templates $0,1,2, \ldots, 9$ in a standard position. The classification was performed by minimum-distance in the space of the ZM magnitudes. Thanks to the invariance of the ZMs and to the numerical stability of the proposed algorithm, all digits were recognized correctly. In Table 1, one can see the distances calculated for several instances of digit 5. This experiment clearly illustrates the performance of the proposed method on real data and its applicability in robot navigation and in similar tasks where one has to recognize patterns independently of their orientation and in a fast and numerically stable way.

Table 1: The classification of digit 5 based on nine Zernike features against the database of the standard position templates $0,1,2, \ldots, 9$ using the minimum-distance rule. The table shows the Euclidean distance in the feature space, the values in bold are the minimum distances.

\begin{tabular}{c|ccccccc}
\hline & \multicolumn{7}{|c}{$\begin{array}{c}\text { Segmented images of Fig. 5 with } \\
\text { different viewing angles }\end{array}$} \\
\hline $\begin{array}{c}\text { Standard } \\
\text { database }\end{array}$ & 5 & 5 & $S$ & $\subseteq$ & $\mathbf{S}$ & $n$ \\
\hline 0 & 0.055374 & 0.054913 & 0.054860 & 0.054974 & 0.054574 & 0.055458 \\
\hline 1 & 0.017219 & 0.017280 & 0.017354 & 0.017559 & 0.016875 & 0.017591 \\
\hline 2 & 0.078091 & 0.078517 & 0.078388 & 0.078555 & 0.079083 & 0.077677 \\
\hline 3 & 0.060124 & 0.060425 & 0.060265 & 0.060317 & 0.060139 & 0.060046 \\
\hline 4 & 0.091344 & 0.091048 & 0.091209 & 0.091031 & 0.091239 & 0.091626 \\
\hline 5 & $\mathbf{0 . 0 0 0 6 4 1}$ & $\mathbf{0 . 0 0 0 4 9 2}$ & $\mathbf{0 . 0 0 1 1 7 6}$ & $\mathbf{0 . 0 0 1 0 2 4}$ & $\mathbf{0 . 0 0 1 2 1 4}$ & $\mathbf{0 . 0 0 1 3 4 3}$ \\
\hline 6 & 0.033656 & 0.033424 & 0.033329 & 0.033202 & 0.033133 & 0.033969 \\
\hline 7 & 0.044682 & 0.045091 & 0.045117 & 0.044652 & 0.045496 & 0.044916 \\
\hline 8 & 0.043058 & 0.042639 & 0.042676 & 0.042818 & 0.042411 & 0.043170 \\
\hline 9 & 0.041933 & 0.041517 & 0.041379 & 0.041684 & 0.040824 & 0.042024 \\
\hline
\end{tabular}



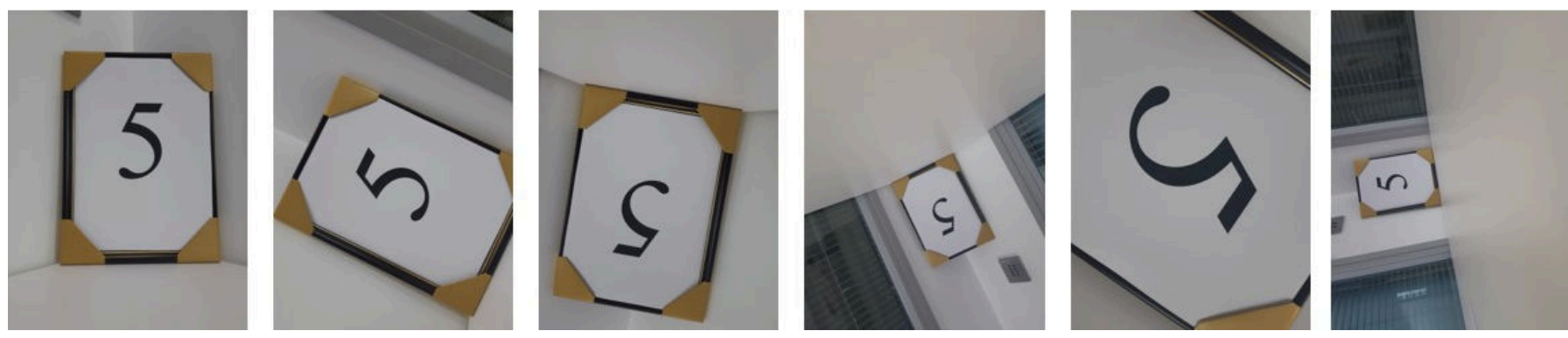

Fig. 5: The indoor scene with the test printed digits.

\section{CONCLUSION}

Different from existing one-variable GF of the radial Zernike polynomials, this paper explores a 2D form of GFs to generate Zernike polynomials. We observed some interesting properties of 2D unilateral Z-transform to derive two-variable GF of the radial Zernike polynomials based on a recurrence relation that depends neither on the degree nor on the azimuthal order of the radial polynomials. Consequently, we develop a two dimensional digital system for systematic generation of the entire orders of Zernike polynomials. With the generated GF, we proposed a spatio-temporal implementation scheme for producing of the radial Zernike polynomials. The experiment clearly shows the performance of the proposed method on real data and its applicability in robot navigation by using ZMs invariant properties.

\section{ACKNOWLEDGMENT}

This work was supported by the Czech Science Foundation (Barmak Honarvar with Grant No. 18-26018Y and Jan Flusser with Grant No. GA18-07247S). It has been also supported by the Praemium Academiae, awarded by the Czech Academy of Sciences, and by the Joint Laboratory SALOME 2.

\section{REFERENCES}

[1] F. Zernike, "Beugungstheorie des Schneidenver-Fahrens und seiner verbesserten Form, der Phasenkontrastmethode," Physica, vol. 1, no. 7-12, pp. 689-704, 1934.

[2] R. Navarro, J. Arines, and R. Rivera, "Direct and inverse discrete Zernike transform,” Opt. Express, vol. 17, no. 26, pp. 24 269-24 281, Dec 2009.

[3] R. Martínez-Cuenca, V. Durán, J. Arines, J. Ares, Z. Jaroszewicz, S. Bará, L. Martínez-León, and J. Lancis, "Closed-loop adaptive optics with a single element for wavefront sensing and correction," Optics letters, vol. 36, no. 18, pp. 3702-3704, 2011.

[4] R. Navarro, J. Arines, and R. Rivera, "Wavefront sensing with critical sampling," Opt. Lett., vol. 36, no. 4, pp. 433-435, Feb 2011.

[5] J. Schwiegerling, "Scaling pseudo-Zernike expansion coefficients to different pupil sizes," Opt. Lett., vol. 36, no. 16, pp. 3076-3078, Aug 2011.

[6] G.-M. Dai, "Scaling Zernike expansion coefficients to smaller pupil sizes: a simpler formula," J. Opt. Soc. Am. A, vol. 23, no. 3, pp. 539-543, Mar 2006.

[7] A. J. Janssen and P. Dirksen, "Computing Zernike polynomials of arbitrary degree using the discrete Fourier transform," Journal of the European Optical Society-Rapid publications, vol. 2, 2007.

[8] C. Kulcsár, H.-F. Raynaud, C. Petit, and J.-M. Conan, "Minimum variance prediction and control for adaptive optics," Automatica, vol. 48, no. 9, pp. 1939 - 1954, 2012
[9] E. Justh, P. Krishnaprasad, and M. Vorontsov, "Analysis of a highresolution optical wave-front control system," Automatica, vol. 40, no. 7, pp. $1129-1141,2004$.

[10] X. Gao, Q. Wang, X. Li, D. Tao, and K. Zhang, "Zernike-momentbased image super resolution," IEEE Transactions on Image Processing, vol. 20, no. 10, pp. 2738-2747, Oct 2011.

[11] Z.-J. Liu, Q. Li, Z.-W. Xia, and Q. Wang, "Target recognition of ladar range images using even-order Zernike moments," Appl. Opt., vol. 51, no. 31, pp. 7529-7536, Nov 2012.

[12] E. C. Kintner, "On the mathematical properties of the Zernike polynomials," Optica Acta, vol. 23, pp. 679-680, 1976.

[13] A. Prata and W. Rusch, "Algorithm for computation of Zernike polynomials expansion coefficients," Applied Optics, vol. 28, no. 4, pp. 749754, 1989.

[14] B. Honarvar and R. Paramesran, "Recursive formula to compute Zernike radial polynomials," Opt. Lett., vol. 38, no. 14, pp. 2487-2489, Jul 2013.

[15] M. Born and E. Wolf, Principles of Optics, 7th ed. Cambridge University Press, 1999.

[16] M. R. Teague, "Image analysis via the general theory of moments," Journal of the Optical Society of America, vol. 70, no. 8, pp. 920-930, 1980.

[17] A. Wallin and O. Kübler, "Complete sets of complex Zernike momen invariants and the role of the pseudoinvariants," IEEE Transactions on Pattern Analysis and Machine Intelligence, vol. 17, no. 11, pp. 11061110, 1995.

[18] H. Wilf, Generatingfunctionology. 2nd Edition, Academic Press, London, 1994.

[19] N. Chitanont, K. Yatabe, K. Ishikawa, and Y. Oikawa, "Spatio-temporal filter bank for visualizing audible sound field by Schlieren method,' Applied Acoustics, vol. 115, pp. 109 - 120, 2017.

[20] J. Flusser, T. Suk, and B. Zitová, 2D and 3D image analysis by moments John Wiley \& Sons, 2016. 
2019-12-19

\section{2-D generating function of the zernike polynomials and their application for image classification}

Honarvar Shakibaei Asli, Barmak

IEEE

Honarvar Shakibaei Asli B, Flusser J, Zhao Y. (2019) 2-D generating function of the zernike polynomials and their application for image classification. In: 2019 Ninth International

Conference on Image Processing Theory, Tools and Applications (IPTA), 6-9 November 2019, Istanbul, Turkey

https://doi.org/10.1109/IPTA.2019.8936089

Downloaded from Cranfield Library Services E-Repository 